\title{
Light mesons in a strong magnetic field on the lattice
}

\section{E.V. Luschevskaya*}

Institute of Theoretical and Experimental Physics, Bolshaya Cheremushkinskaya 25, Moscow, 117218, Russia

E-mail: luschevskaya@itep.ru

\section{O.V. Teryaev}

Joint Institute for Nuclear Research, Dubna, Russia

E-mail: teryaevetheor.jinr.ru

\section{O.A. Kochetkov}

Institute of Theoretical and Experimental Physics, Bolshaya Cheremushkinskaya 25, Moscow, 117218, Russia

Institut fur Theoretische Physik, Universitat Regensburg, D-93040 Regensburg, Germany

E-mail: oleg.kochetkov@physik.uni-r.de

\begin{abstract}
We explore the masses (ground states energies) of neutral and charged $\rho$ mesons in strong abelian magnetic field in $S U(3)$ gluodynamics. The energies of these particle in the external magnetic field depends on its spin projection $s_{z}$ on the axis of external magnetic field. The masses of $\rho^{0}$ meson with $s_{z}= \pm 1$ increase with the field. The masses of $\rho^{ \pm}$mesons with zero spin also grow with the magnetic field. The ground state energies of $\rho^{-}$meson with $s_{z}=-1$ and $\rho^{+}$meson with $s_{z}=+1$ decrease as a function of the field, while the energies of $\rho^{+}$meson with $s_{z}=-1$ and $\rho^{-}$ meson with $s_{z}=+1$ rise with the field value.
\end{abstract}

XXII International Baldin Seminar on High Energy Physics Problems,

15-20 September 2014

JINR, Dubna, Russia

${ }^{*}$ Speaker. 


\section{Introduction}

We investigate the dependence of ground energy states of light mesons depending on the magnetic field of hadronic scale. This study is of interest because it can give an indication of the phase structure of QCD in a strong magnetic field. Now it is possible to observe such magnetic fields in non-central heavy-ion collisions in terrestrial laboratories (ALICA, RHIC, NICA, FAIR). The field value can reach up to $15 m_{\pi}^{2} \sim 0.27 \mathrm{GeV}^{2}$ [1], i.e. the order of energies at which the QCD effects appear. The strong magnetic fields of $\sim 2 \mathrm{GeV}$ may be associated with the formation of the Early Universe [2]. Quantum chromodynamics in a strong magnetic field shows a lot of bright interesting effects which have been observed in experiment and discovered theoretically, for example inverse magnetic catalysis [3], chiral magnetic effect [4, 5, 6, 7, 8], enhancement of the chiral symmetry breaking $[9,10,11,12,13]$.

The investigations related to QCD phase diagram in strong magnetic field are presented in $[14,15,16,17,18,19,20,21]$. Numerical simulations in QCD with $N_{f}=2$ and $N_{f}=2+1$ show that strongly interacting matter in strong magnetic field posses paramagnetic properties in the confinement and deconfinement phases [22, 23, 24].

In this work we explore the splitting of ground state energy of neutral $\rho^{0}$ and charged vector mesons $\rho^{ \pm}$and neutral axial meson $a_{1}^{0}$ depending on its spin projection on the axis of the external abelian magnetic field. This exploration is important because such splitting can lead to the asymmetry of emitted neutral and charged particles above and under reaction plane and contribute to the chiral magnetic effect.

Articles [25, 26, 27, 28] and [29] are also devoted to the behaviour of hadron masses in the external abelian magnetic field. The magnetic moments of mesons have been explored in [30].

\section{Details of calculations}

The technical details of our calculations are presented in [31]. The tadpole improved Symanzik action was used for the generation of $S U(3)$ statistically independent lattice gauge configurations. Then we solve Dirac equation numerically

$$
D \psi_{k}=i \lambda_{k} \psi_{k}, \quad D=\gamma^{\mu}\left(\partial_{\mu}-i A_{\mu}\right)
$$

and find eigenfunctions $\psi_{k}$ and eigenvectors $\lambda_{k}$ for test quark in the external gauge field $A_{\mu}$.

We investigate the behaviour of the ground energy states in background gauge field, which is a sum of non-abelian $S U(3)$ gluonic field and $U(1)$ abelian uniform magnetic field. Abelian gauge fields interact only with quarks. In our calculations we do not consider dynamical quarks. We add the magnetic field only in Dirac operator. Our simulations has been carried out on symmetrical lattices with lattice volumes $16^{6}$ and $18^{4}$ and lattice spacings $a=0.115 \mathrm{fm}$ and $0.125 \mathrm{fm}$ respectively.

We calculate the following observables in coordinate space and background gauge field $A$

$$
\left\langle\psi^{\dagger}(x) O_{1} \psi(x) \psi^{\dagger}(y) O_{2} \psi(y)\right\rangle_{A},
$$

where $O_{1}, O_{2}=\gamma_{5}, \gamma_{5} \gamma_{\mu}, \gamma_{\mu}$ are Dirac gamma matrices, $\mu, v=1, . ., 4$ are Lorenz indices. 
In order to calculate the observables (2.2) we calculate quark propagators in coordinate space. For $M$ lowest eigenstates a massive Dirac propagator is represented by the following sum:

$$
D^{-1}(x, y)=\sum_{k<M} \frac{\psi_{k}(x) \psi_{k}^{\dagger}(y)}{i \lambda_{k}+m} .
$$

In our calculations we use $M=50$. For the observables (2.2) the following equation is fulfilled

$$
\left\langle\bar{\psi} O_{1} \psi \bar{\psi} O_{2} \psi\right\rangle_{A}=-\operatorname{tr}\left[O_{1} D^{-1}(x, y) O_{2} D^{-1}(y, x)\right]+\operatorname{tr}\left[O_{1} D^{-1}(x, x)\right] \operatorname{tr}\left[O_{2} D^{-1}(y, y)\right] .
$$

We calculate the correlators and make its Fourier transformation. As we are interested in the meson ground state, we choose $\langle\mathbf{p}\rangle=0$. For particles with zero momentum their energy is equal to mass $E_{0}=m_{0}$.

The expansion of correlation function to exponential series has the form

$$
\tilde{C}\left(n_{t}\right)=\left\langle\psi^{\dagger}\left(\mathbf{0}, n_{t}\right) O_{1} \psi\left(\mathbf{0}, n_{t}\right) \psi^{\dagger}(\mathbf{0}, 0) O_{2} \psi(\mathbf{0}, 0)\right\rangle_{A}=\sum_{k}\left\langle 0\left|O_{1}\right| k\right\rangle\left\langle k\left|O_{2}^{\dagger}\right| 0\right\rangle e^{-n_{t} a E_{k}},
$$

where $a$ is the lattice spacing, $n_{t}$ is the number of nodes in the time direction, $E_{k}$ is the energy of the state. From expansion (2.5) one can see that for large $n_{t}$ the main contribution origins from the ground state. Because of periodic boundary conditions the main contribution to the ground state has the following form

$$
\tilde{C}_{f i t}\left(n_{t}\right)=A_{0} e^{-n_{t} a E_{0}}+A_{0} e^{-\left(N_{T}-n_{t}\right) a E_{0}}=2 A_{0} e^{-N_{T} a E_{0} / 2} \cosh \left(\left(N_{T}-n_{t}\right) a E_{0}\right),
$$

where $A_{0}$ is a constant, $E_{0}$ is the energy of the ground state.

Mass of the ground state can be evaluated fitting the correlator (2.5) with (2.6) function. In order to minimize the errors and exclude the contribution of excited states we take various values of $n_{t}$ from the interval $5 \leq n_{t} \leq N_{T}-5$. Masses of $\rho$ mesons have been obtained from correlator (2.2), where $O_{1}, O_{2}=\gamma_{\mu}$. For $O_{1}, O_{2}=\gamma_{5} \gamma_{\mu}$ we get axial-vector $a_{1}^{0}$ meson.

\section{Neutral mesons}

The correlation functions for various spatial directions are given by the following relations

$$
\begin{aligned}
& C_{x x}^{V V}=\left\langle\bar{\psi}\left(\mathbf{0}, n_{t}\right) \gamma_{1} \psi\left(\mathbf{0}, n_{t}\right) \bar{\psi}(\mathbf{0}, 0) \gamma_{1} \psi(\mathbf{0}, 0)\right\rangle, \\
& C_{y y}^{V V}=\left\langle\bar{\psi}\left(\mathbf{0}, n_{t}\right) \gamma_{2} \psi\left(\mathbf{0}, n_{t}\right) \bar{\psi}(\mathbf{0}, 0) \gamma_{2} \psi(\mathbf{0}, 0)\right\rangle, \\
& C_{z z}^{V V}=\left\langle\bar{\psi}\left(\mathbf{0}, n_{t}\right) \gamma_{3} \psi\left(\mathbf{0}, n_{t}\right) \bar{\psi}(\mathbf{0}, 0) \gamma_{3} \psi(\mathbf{0}, 0)\right\rangle .
\end{aligned}
$$

The form of the density matrix for vector particle with spin $s=1$ gives the formulas for obtaining the energies of meson with various spin projections on the axis of the external magnetic field. For the $s_{z}=0$ one can obtain the energy of the ground state from the $C_{z z}^{V V}$ correlator. The combinations of correlators

$$
C^{V V}\left(s_{z}= \pm 1\right)=C_{x x}^{V V}+C_{y y}^{V V} \pm i\left(C_{x y}^{V V}-C_{y x}^{V V}\right) .
$$

gives the energies of mesons with $s_{z}=+1$ and $s_{z}=-1$. 


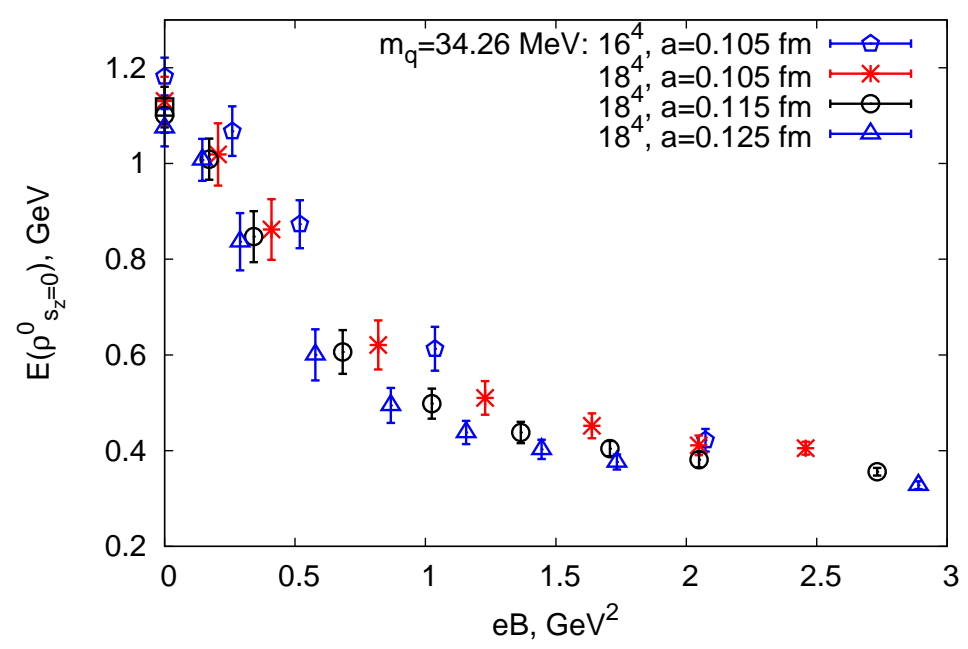

Figure 1: The ground state energy of the neutral $\rho^{0}$ meson with spin $s_{z}=0$ as a function of magnetic field for lattice volume $16^{4}$, lattice spacing $0.105 \mathrm{fm}$ and lattice volume $18^{4}$, lattice spacings $a=0.105 \mathrm{fm}$, $0.115 \mathrm{fm}, 0.125 \mathrm{fm}$ and bare quark mass $34.26 \mathrm{MeV}$.

The decreasing mass of neutral vector meson with zero spin is shown in Fig.1. For the case of neutral mesons we consider degenerate $d$ and $u$ quarks, so the mixing between $\rho^{0}\left(s_{z}=0\right)$ and $\pi^{0}(s=0)$ states doesn't occur on the lattice. One know that in the presence of strong magnetic field the branching in the decay $\rho \rightarrow \pi \gamma$ has to be large.

In Fig. 2 we depict the mass of the $\rho^{0}$ meson with spin projections $s_{z}= \pm 1$. The masses for $s_{z}=-1$ and $s_{z}=+1$ are equal which is a manifestation of C-parity of $\rho^{0}$ meson. They increase with the field value. The lattice spacing and lattice volume artifacts are small. We make a fit

$$
E=E_{0}(B=0)-2 \pi \beta_{m}^{|s|=1}(e B)^{2}
$$

to the data on the interval $e B \in\left[0,2.2 \mathrm{GeV}^{2}\right]$, where $\beta_{m}^{|s|=1}$ is the magnetic polarizability of meson with spin $\left|s_{z}\right|=1$. All the fits correspond to the $18^{4}$ lattice, the solid line is for the lattice spacing $0.105 \mathrm{fm}$, the dashed line is for the lattice spacing $0.115 \mathrm{fm}$ and dashed-dotted line corresponds to the $a=0.125 \mathrm{fm}$. We observe the square-law dependence of the energy versus $e B$. One can calculate the magnetic polarizability of neutral $\rho^{0}$ meson from such fits, but the extrapolation to zero lattice spacing is necessary because the $\beta_{m}^{|s|=1}$ value is sensitive to the lattice spacing value. We will calculate the magnetic polarizability in the next work.

For the case of nonzero spin $\beta_{m}^{|s|=1}$ value is negative and it means that the external magnetic field shrinks the wave function of the $\rho^{0}$ meson. In case of zero meson spin projection on the field axis the magnetic polarizability is positive (one can see this from Fig.1) and it means that the magnetic field enlarge the size of $\rho^{0}$ wave function.

In Fig. 3 we present the mass of axial meson $a_{1}^{0}$ with spin projection $\left|s_{z}\right|=1$. Like in the $\rho$ meson case we make a fit (3.5) to the data but at larger interval $e B \in\left[0,4.5 \mathrm{GeV}^{2}\right]$, its value is also negative. The energy increase quadratically. For the case of axial-vector $a_{1}^{0}$ meson the large statistics and large lattices are needed. 


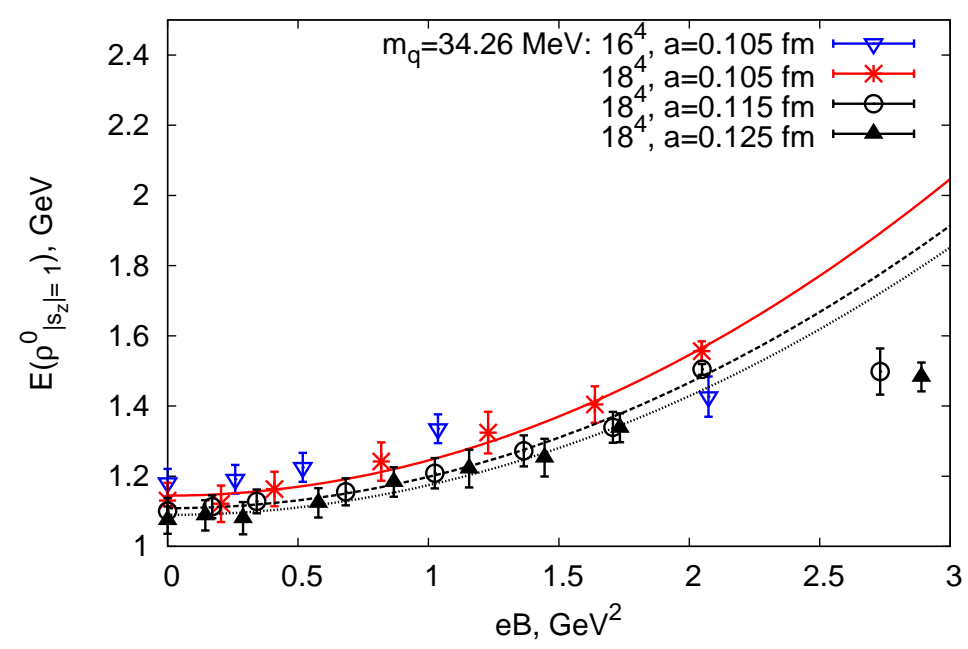

Figure 2: The energy of the ground state of vector neutral $\rho^{0}$ meson obtained from the cosh. fits to the correlators $C_{s_{z}= \pm 1}^{V V}$ as a function of the magnetic field together with the fits $E=E_{0}(B=0)-2 \pi \beta_{m}^{|s|=1}(e B)^{2}$ to the data on the interval $e B \in\left[0,2.2 \mathrm{GeV}^{2}\right]$. All the fits correspond to the $18^{4}$ lattice, the solid line is for the lattice spacing $0.105 \mathrm{fm}$, the dashed line is for the lattice spacing $0.115 \mathrm{fm}$ and dashed-dotted line corresponds to the $a=0.125 \mathrm{fm}$.

\section{Charged mesons}

In our case the energy levels of free charged pointlike particle in a background magnetic field is described by the formula

$$
E^{2}=|q B|-g s_{z} q B+m^{2}(B=0),
$$

here $g$-factor characterizes magnetic properties of the particle, $q$ is the charge of the particle, $m^{2}(B=0)$ is the particle mass at $B=0$. The values of $g$ and $m^{2}(B=0)$ can be obtained from the fitting to the data. The (4.1) is true only for point-like particle and doesn't take into account polarizabilities of mesons. We cannot distinguish negative and positive vector $\rho$ mesons on the lattice because the exchange of the particle charge to the opposite is equivalent to the exchange of $B$ to $-B$ which gives the same masses at $s_{z}=0$.

In Fig.4 we represent the energy of the charged vector $\rho$ meson with $s_{z}=0$. The data is in accordance with formula (4.1) at $e B \in\left[0,2 \mathrm{GeV}^{2}\right]$. We make a fit $E=\sqrt{a+e B}$ to the data for lattice volume $18^{4}$ and lattice spacing $a=0.105 \mathrm{fm}$, where $a=m^{2}(B=0)$ is the fit parameter.

We represent the energies of the $\rho^{-}$with spin projections $s_{z}=-1$ and $s_{z}=+1$ in Figs. 5 and 6 correspondingly. The energy of the $\rho^{-}$ground state with $s_{z}=-1$ decreases with the field value and the data agree with the fit $E=\sqrt{(1-a) e B+b}$ at $e B \in\left[0,0.6 \mathrm{GeV}^{2}\right]$. Possibly the reason of the discrepancy between the data and the fit is non-zero polarizability of charged $\rho$ meson. There is some effect which prevents the appearance of the tachyonic mode. The energy of $\rho^{-}$ground state with $s_{z}=+1$ increases with the field value. The function $E=\sqrt{(1+a) e B+b}$ gives the excellent fits for the all presented data. Simple estimates give the following values of the magnetic field corresponding to the cut-off: $2.9 \mathrm{GeV}^{2}$ for lattice spacing $a=0.115 \mathrm{fm}$ and $2.5 \mathrm{GeV}^{2}$ for $a=0.125 \mathrm{fm}$. 


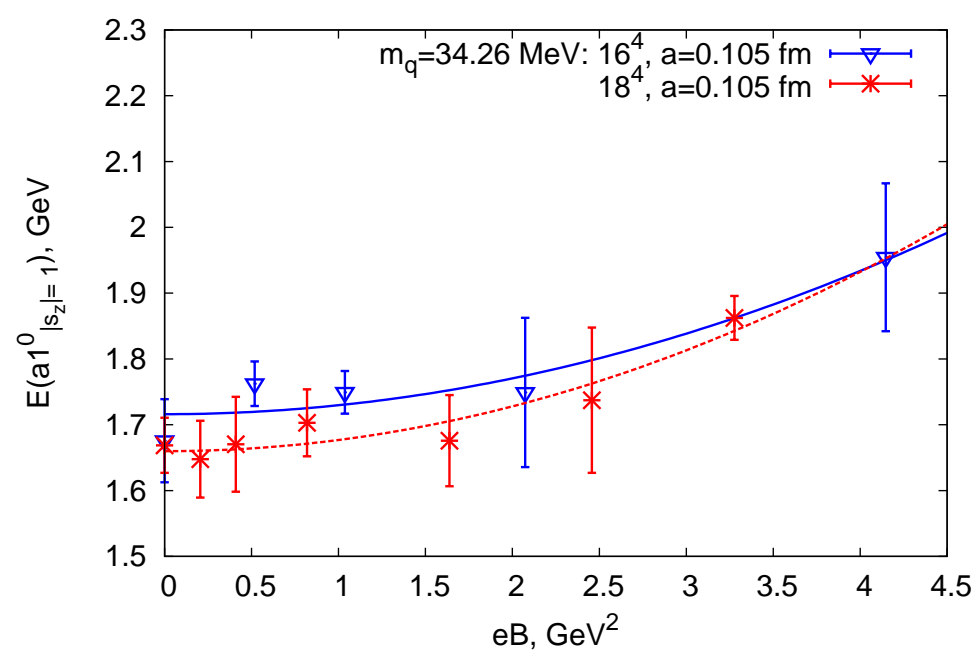

Figure 3: The mass of axial meson $a_{1}^{0}$ with spin $\left|s_{z}\right|=1$ as a function of magnetic field for lattice volumes $16^{4}, 18^{4}$, lattice spacings $a=0.105 \mathrm{fm}$ and bare quark mass $34.26 \mathrm{MeV}$. The solid line correspnds to the fit $E=E_{0}(B=0)-2 \pi \beta_{m}^{|s|=1}(e B)^{2}$ to the data for $16^{4}$ lattice, the dashed line is for $18^{4}$ lattice.

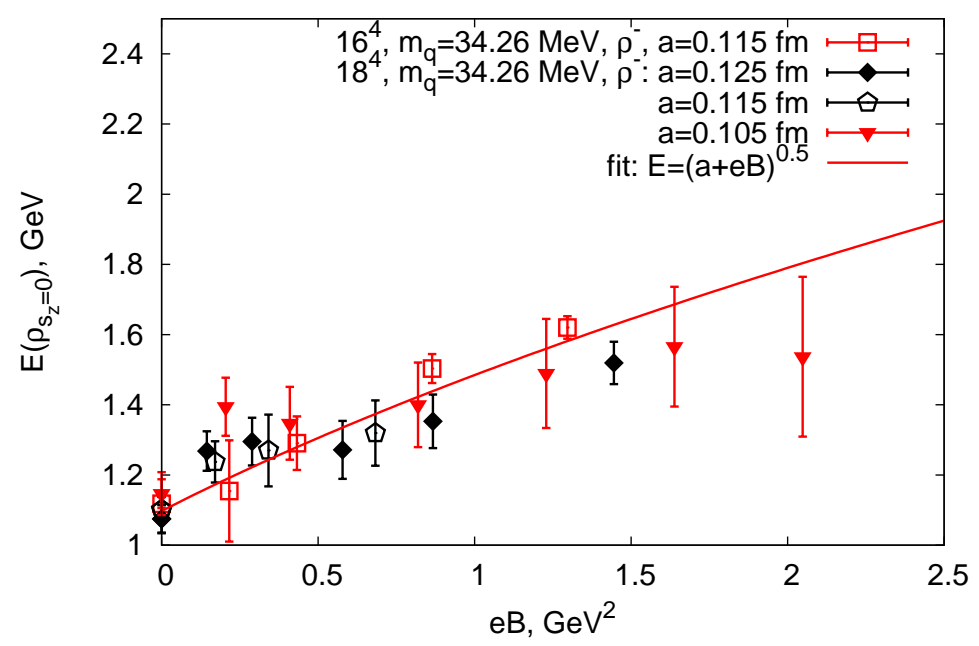

Figure 4: The ground state energy of the charged vector meson $\rho^{-}$with zero spin $s_{z}=0$ as a function of magnetic field for $16^{4}$ and $18^{4}$ lattices, $a=0.105$, fm, $a=0.115$, fm and $0.125 \mathrm{fm}$ and various bare quark masses.

\section{Conclusions}

We explore the ground state energies the neutral $\rho^{0}, a_{1}^{0}$ mesons and charged $\rho^{ \pm}$mesons in $S U$ (3) lattice gauge theory. The energies of neutral mesons with non-zero spin $\left|s_{z}\right|=1$ increase while the energies of neutral particles with zero spin $s_{z}=0$ decrease. For the case of nonzero spin the data demonstrate square-law dependence on field value $e B$.

The energy splitting of charged particles is described by Landau levels at moderate magnetic field: for $s=0$ till $e B=2 \mathrm{GeV}^{2}$, for $\rho^{-}\left(s_{z}=+1\right)\left(\rho^{+}\left(s_{z}=-1\right)\right)$ at all considered in this work 


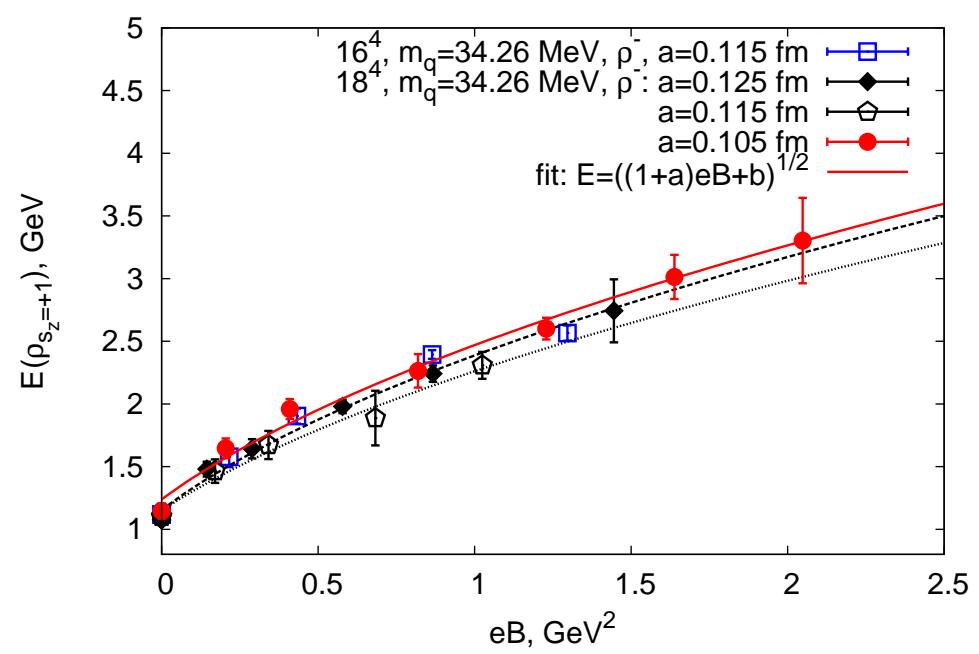

Figure 5: The ground state energy of $\rho^{-}$meson with spin $s_{z}=-1$ versus the field value for lattice volumes $16^{4}$ and $18^{4}$, lattice spacings $a=0.105 \mathrm{fm}, a=0.115 \mathrm{fm}$ and $0.125 \mathrm{fm}$ and various bare quark masses. Fits are made for the $18^{4}$ lattice volume; solid line corresponds to the $a=0.105 \mathrm{fm}$ lattice spacing, dot dashed line is for $0.115 \mathrm{fm}$, dashed line is for $a=0.125 \mathrm{fm}$.

magnetic fields and for $\rho^{-}\left(s_{z}=-1\right)\left(\rho^{+}\left(s_{z}=+1\right)\right)$ at $e B \in\left[0,0.6 \mathrm{GeV}^{2}\right]$. We didn't observe any evidence in favour of charged vector meson condensation as presented in [33, 34]. There is some mechanism which prevents the appearance of tachyonic mode.

\section{Acknowledgments}

This work was carried out with the financial support of Grant of President MK-6264.2014.2, FRRC grant of Rosatom SAEC and Helmholtz Assotiation and RFBR grant 14-02-00395 A. The authors are grateful to FAIR-ITEP supercomputer center where these numerical calculations were performed. O.T. is supported by RFBR grants 12-02-00613, 14-01-00647 and in part by HeisenbergLandau program.

\section{References}

[1] V. Skokov, A. Illarionov and V. Toneev, Int. J. Mod. Phys. A 245925 (2009), arXiv: 0907.1396[nucl-th]

[2] D. Grasso and H.R. Rubinstein Phys. Rept. 348163 (2001), arXiv: astro-ph/0009061

[3] F. Bruckmann, G. Endrodi and T. Kovacs, JHEP 04112 (2013), arXiv: 1303.3972 [hep-lat]

[4] S.A. Voloshin, Phys. Rev. C 70057901 (2004), arXiv: hep-ph/0406311.

[5] S.A. Voloshin, arXiv: 0806.0029 [nucl-ex]; H. Caines, arXiv: 0906.0305 [nucl-ex].

[6] D.E. Kharzeev, L.D. McLerran, and H.J. Warringa, Nucl. Phys. A 803227 (2008), arXiv: 0711.0950; K. Fukushima, D.E. Kharzeev, and H.J. Warringa, Phys. Rev. D 78074033 (2008), arXiv: 0808.3382; H.J. Warringa, arXiv: 0906.2803; D.E. Kharzeev, arXiv: 0906.2808 and arXiv: 0908.0314. 


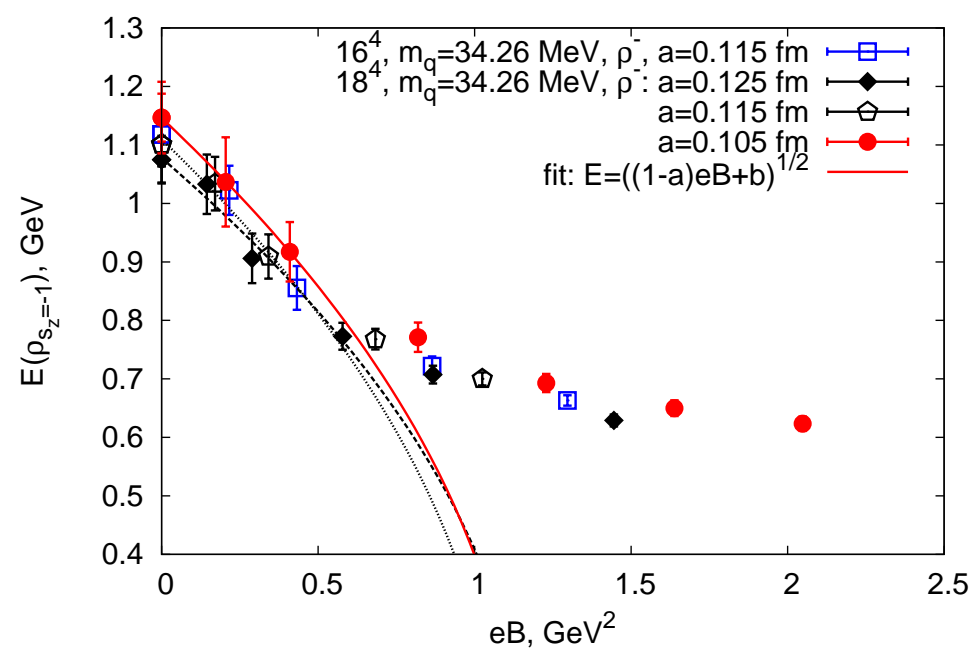

Figure 6: The ground state energy of $\rho^{-}$meson with spin $s_{z}=+1$ versus the field value for lattice volumes $16^{4}$ and $18^{4}$, lattice spacings $a=0.105 \mathrm{fm}, a=0.115 \mathrm{fm}$ and $0.125 \mathrm{fm}$ and various bare quark masses. Fits are made for the $18^{4}$ lattice volume; solid line corresponds to the $a=0.105 \mathrm{fm}$ lattice spacing, dot dashed line is for $0.115 \mathrm{fm}$, dashed line is for $a=0.125 \mathrm{fm}$.

[7] P.V. Buividovich, M.N. Chernodub, E.V. Luschevskaya, M.I. Polikarpov, Phys. Rev. D 80054503 (2009), arXiv:0907.0494 [hep-lat].

[8] P.V. Buividovich, M.N. Chernodub, E.V. Luschevskaya, M.I. Polikarpov, PoSLAT2009 080 (2009), arXiv:0910.4682 [hep-lat].

[9] V.P. Gusynin, V.A. Miransky and I.A. Shovkovy, Nucl. Phys. B 462 249-290 (1996), hep-ph/9509320.

[10] S. P. Klevansky and R. H. Lemmer, Phys. Rev. D 393478 (1989);

[11] D. Ebert, K. G. Klimenko, M. A. Vdovichenko and A. S. Vshivtsev, Phys. Rev. D 61025005 (1999), hep-ph/9905253.

[12] E. S. Fraga and A. J. Mizher, Phys. Rev. D 78025016 (2008), arXiv: 0804.1452; Nucl. Phys. A 820 103C (2009), arXiv: 0810.3693.

[13] A. Goyal, M. Dahiya, Phys. Rev. D 62025022 (2000), hep-ph/9906367.

[14] M. D’Elia, S. Mukherjee, F. Sanfilippo, Phys. Rev. D 82051501 (2010), arXiv: 1005.5365v2 [hep-lat].

[15] G.S. Bali, F. Bruckman, G. Endrodi, Z. Fodor, S.D. Katz, S. Krieg, A. Schaefer, K.K. Szabo, JHEP 02 044 (2012), arXiv: 1111.4956 [hep-lat].

[16] E. S. Fraga and A. J. Mizher, Phys. Rev. D 78025016 (2008), arXiv: 0804.1452; Nucl. Phys. A 820 103C (2009), arXiv: 0810.3693; A.J. Mizher, M.N. Chernodub, and E.S. Fraga, Phys. Rev. D 82 105016 (2010).

[17] R. Gatto and M. Ruggieri, Phys. Rev. D 83034016 (2011), arXiv: 1012.1291; R. Gatto and M. Ruggieri, Phys. Rev. D 82054027 (2010), arXiv: 1007.0790;

[18] K. Kashiwa, Phys. Rev. D 83117901 (2011), arXiv: 1104.5167.

[19] S. Kanemura, H.-T. Sato, H. Tochimura, Nucl. Phys. B 517567 (1998), arXiv: hep-ph/9707285. 
[20] K.G. Klimenko, Theor. Math. Phys. 901 (1992).

[21] V.G. Bornyakov, P.V. Buividovich, N. Cundy, O.A. Kochetkov and A. Schaefer Phys. Rev. D 90 034501 (2014), arXiv: 1312.5628 [hep-lat]

[22] C. Bonatti, M. D’Elia, M. Mariti, F. Negro, F. Sanfilippo, PoS Lattice (2013), arXiv:1312.5070.

[23] C. Bonatti, M. D’Elia, M. Mariti, F. Negro, F. Sanfilippo, arXiv: 1310.8656.

[24] G.S. Bali, F. Bruckman, G. Endrodi, A. Schaefer, PoS Lattice (2013), arXiv: 1310.8145.

[25] M.A. Andreichikov, B.O. Kerbikov, V.D. Orlovsky, Yu.A. Simonov, Phys. Rev. D 87094029 (2013), arXiv: 1304.2533 [hep-ph].

[26] M.A. Andreichikov, B.O. Kerbikov, V.D. Orlovsky, Yu.A. Simonov, Phys. Rev. D 89074033 (2014), arXiv: 1312.2212 [hep-ph].

[27] V.D. Orlovsky, Yu.A. Simonov, JHEP 1309136 (2013), arXiv:1306.2232 [hep-ph].

[28] Y. Hidaka, A. Yamamoto, Phys. Rev. D 87094502 (2013), arXiv:1209.0007 [hep-ph].

[29] H. Liu, L. Yu and M. Huang, arXiv:1408.1318 [hep-ph]

[30] A.M. Badalian and Yu.A. Simonov, Phys. Rev. D 87074012 (2013), arXiv:1306.2232 [hep-ph].

[31] E.V. Luschevskaya, O.V. Larina, it Nucl. Phys. B 8841 (2014), arXiv: 1203.5699 [hep-lat] .

[32] M.H. Al-Hashimi and U.J. Wiese, Annals Phys. 324343 (2009), arXiv: 0807.0630 [quant-ph].

[33] M.N. Chernodub Phys. Rev. D 82085011 (2010), arXiv: 1008.1055 [hep-ph]

[34] M.N. Chernodub Phys. Rev. Lett. 106142003 (2011), arXiv: 1101.0117v2 [hep-ph] 